\title{
Liver Rejection and Its Differentiation from Other Causes of Graft Dysfunction
}

\author{
CARLOS O. ESQUIVEL, M.D., Ph.D., RONALD JAFFE, M.B., B.Ch., \\ ROBERT D. GORDON, M.D., SHUNZABURO IWATSUKI, M.D., \\ BYERS W. SHAW Jr., M.D., and THOMAS E. STARZL, M.D., Ph.D.
}

Following liver transplantation, a series of events may take place that require rapid assessment and proper treatment in order to achieve good results (Table 1 ). Immediately after transplantation, an slevation in the transaminases occurs that represents ischemic injury during the harvesting of the liver, during its placement in the recipient, or both. The transaminases are in most cases less than 1000 $\mathrm{IU} / \mathrm{ml}$, but may increase as high as $3000 \mathrm{IU} / \mathrm{ml}$, depending on the extent of ischemic injury. Higher values than 3000 are certainly worrisome, since the extent of the damage may be so severe that the liver may not recover. The bilirubin, on the other hand, is usually lower than the preoperative levels due to a "wash out" phenomenon with the use of large quantities of fluid during the operation. In this situation in which multiple transfusions of red blood cells, platelets, plasma, and crystalloid are required, the ischemic injury may not become apparent until the second or third postoperative day when the transaminases reach the highest level.

Approximately one-third of the patients have no further abnormalities of hepatic function, and in these cases the enzymes progressively return to normal. The rest of the patients, representing roughly two thirds, exhibit some manifestations of ongoing hepatic dysfunction, and, although mild in most cases, the abnormalities can lead to graft failure and sometimes death of the patient. We will discuss these complications in more detail.

From the Departments of Surgery and Pathology. University of Pittsburgh Health Center, University of Pittsburgh, Pittsburgh, Pennsylvania.

Reprint requests: Dr. Esquivel, Dept. of Surgery, 3601 Fifth Ave., Room 103, Falk Clinic, Pittsburgh, PA 15213.
TABLE 1. Causes of Graft Dysfunction Following Orthotopic Liver Transplantation

1. Rejection

2. Harvesting-induced injury

3. Vascular thrombosis

4. Infection

5. Bile duct complications

6. Hemolysis

7. Drug-induced hepatic injury

\section{REJECTION}

Rejection is the most common cause of graft dysfunction. It is seen most often during the first three postoperative months, although it can occur at any time. Evidence of rejection is often noticed during the second or third week, but it has been documented as early as the third day after operation. The severity as well as the clincial picture are variable. Many patients become depressed and complain of general malaise and lack of appetite. They may develop fever and abdominal pain. The graft may become firm and tender to palpation. ${ }^{1}$ Elevations may occur in the transaminases, bilirubin, and alkaline phosphatase. Liver biopsy at this stage may help clarify the differential diagnosis. With early rejection, the portal tracts are infiltrated with cells that are largely lymphoid with a minor admixture of neutrophils and eosinophils. Bile ducts and particularly the ductules show cytoplasmic vacuolation and the presence of inflammatory cells (Fig. 1). Rejection may develop with slow and progressive graft dysfunction, or it may present acutely in a more aggressive form, characterized by a rapid increase in the bilirubin and transaminases. The latter is more 

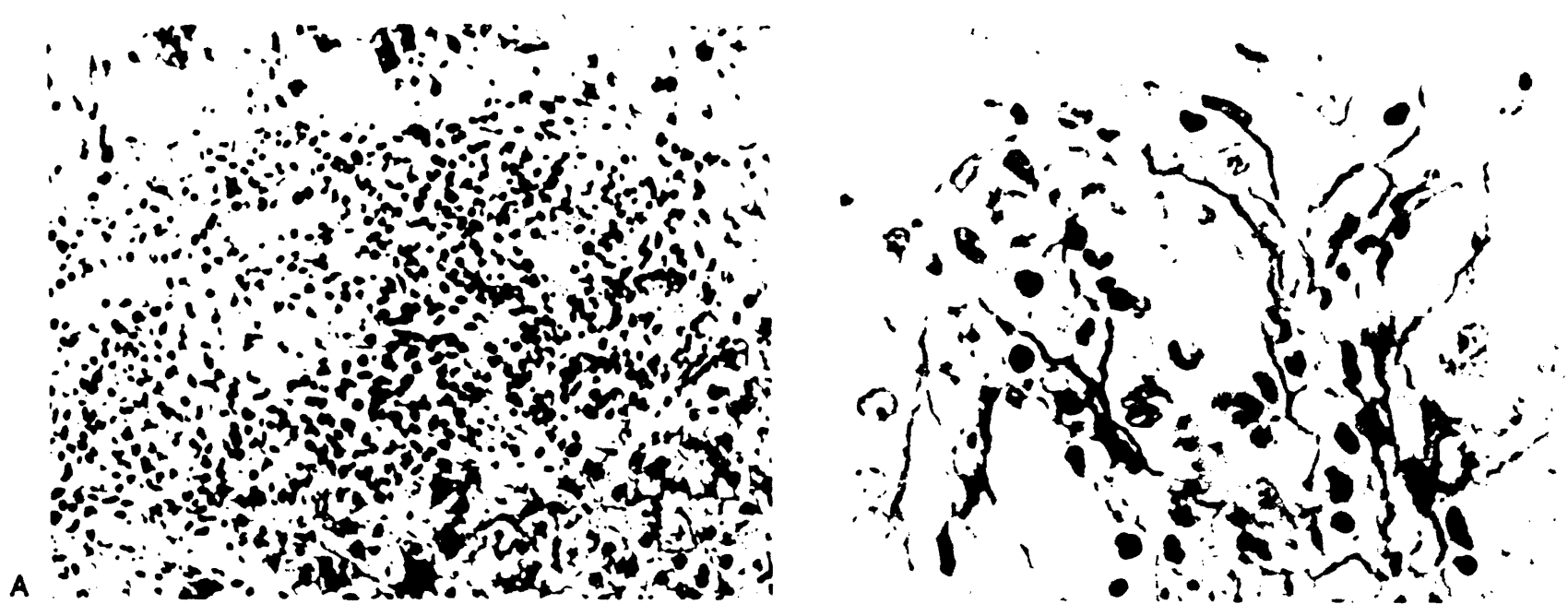

FIG. 1. Early rejection. A: The portal ares is expanded by an infitrete, predominantly tymphoid. Bile ductules appear more cellular than normal. (Trichrome, $x$ 197.) B: The bile ductules are surrounded by lymphocytes, a few of which have breached the basement membrane. The cells are swollen and vacuolated. Hepatocytes at the limiting plate are, at this stage, unaffected. (PAS-diastase, $\times 470.1$

difficult to treat and, frequently, retransplantation is the only method of therapy.

As described elsewhere in this issue of Seminars, treatment consists of increasing doses of steroids, the administration of monoclonal antilymphocyte globulin (ALG), or both. The dosage of steroids depends in part on the size of the patient and the severity of the rejection. For example, in an adult with mild rejection, $1 \mathrm{gm}$ of prednisolone intravenously may suffice, but if the rejection is more severe, the pulse of steroids should be followed by a 5-day burst of prednisolone or prednisone, starting at $200 \mathrm{mg}$ and stopping at a maintenance dose of $20 \mathrm{mg} /$ day. A similar situation in children will be treated with $1 \mathrm{gm}$ of hydrocortisone followed by a tapering dose of prednisolone over 5 days, starting at $100 \mathrm{mg}$ and stopping with a maintenance dose of 20 or $10 \mathrm{mg} /$ day. If rejection cannot be reversed, then a 10- or 14-day course with monoclonal ALG is indicated.

A disturbing combination of problems is that of cytomegalovirus (CMV) infection in the presence of rejection, since the goal of therapy is completely different with these two complications. Reduction of immunosuppression is indicated for a CMV infection, whereas an increase of immunosuppression is required to control rejection. In fact, the most common sequence of events is to have a patient who has been treated for an episode of rejection then develop CMV infection. A liver biopsy at this time may show the presence of intranuclear or intracytoplasmic inclusion bodies as well as the feature characteristic of rejection (Fig. 2). It has been possible to treat the majority of these patients by decreasing maintenance immunosuppression and by giving well-timed pulses of steroids. This problem may be made easier in the future, since new drugs for CMV infection are under investigation at the present time.

\section{ISCHEMIC INJURY}

The causes of ischemic injury during the harvesting or in the recipient's operation or both are listed in Table 2. Initially, there is little trouble in making the diagnosis. There is marked and progresgressive elevation of the bilirubin, and serum transaminase increases are always in the thousands. Severe coagulopathy, hypoglycemia, and renal failure are also observed. The patients exhibit varying degrees of encephalopathy.

Liver biopsy at this stage may distinguish between rejection (Fig. 1) and the features of harvesting-related injury (Fig. 3). A word of caution is in order. The features of early vascular injury may be peripheral and subcapsular in distribution so that a superficial biopsy may not be representative of the entire liver and may provide a pessimistic histologic picture. On occasion, liver function may recover; however, retransplantation is the only hope in the majority of these patients. The 1-year survival rate following retransplantation for ischemic injury is only $20 \%$ compared with $60 \%$ for chronic rejection or arterial thrombosis.

If it is decided, as an option to retransplantation, to hope for recovery from ischemic damage, the supervention of rejection is always a possibility and one that cannot be assessed easily, even with serial biopsies and the most careful attention to liver function tests. 


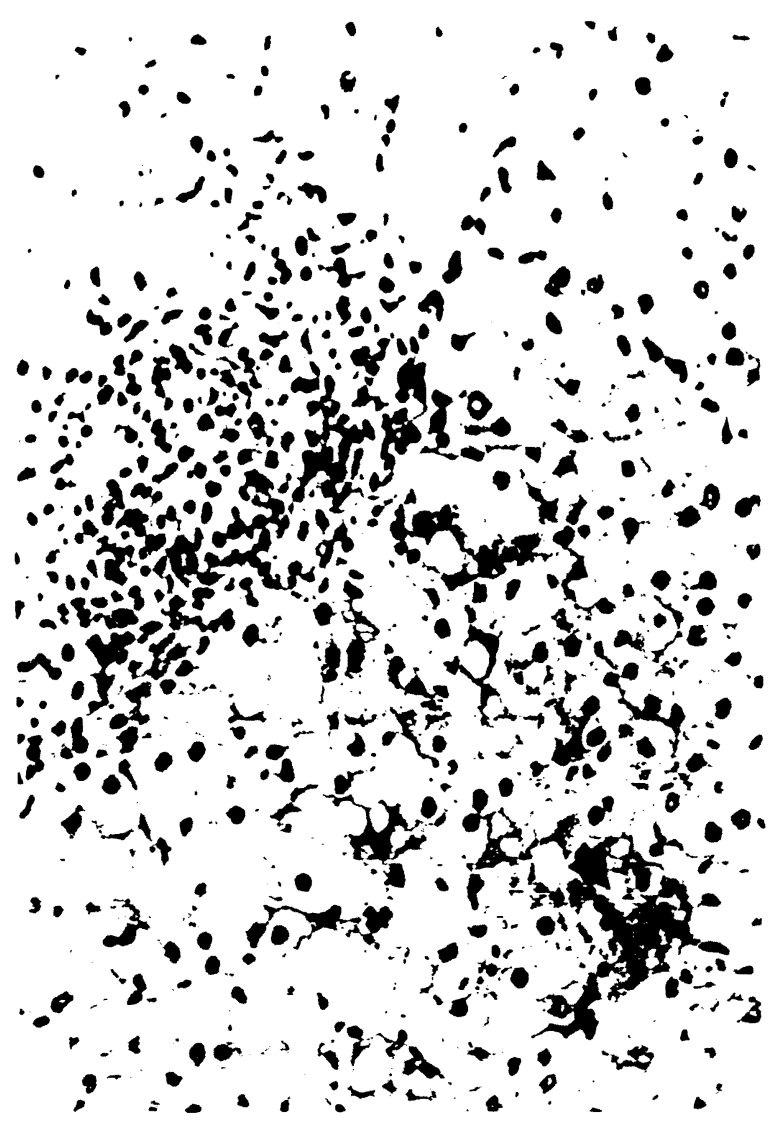

FIG. 2. Cytomegalovirus hepatitis. A: CMV can be presented in the presence of portal features of rejection. The CMV (arrows) is not responsible for the portal cells, often being seen without any portal infiltrate. (H\&E, $\times$ 197.) B: Infected cells have the nuclear inclusion but just as characteristic is the glossy cytoplasm with bright eosinophilic granular inclusions (H\&E, $x$ 470.) C: Tiny neutrophilic microabscesses in the hepatic lobule are often the first clue to the presence of cytomegalovirus (CMV) which may be sparse. (H\&E, $\times 470$.$) Enzyme-antibody reaction$ using a commercially available antibody to CMV reveals a cytoplasmic antigen in the infected cells (inset). (Peroxidase-DAB, $\times 470.1$

\section{VASCULAR THROMBOSIS}

Thrombosis of the hepatic artery is a common cause of graft dysfunction particularly in children. ${ }^{2}$ The incidence is approximately 1.5 and $11.8 \%$ in adults and children, respectively. Arterial thrombosis is the second most common cause for retransplantation in the pediatric populatoin.

Many patients develop a temperature spike, usually $39^{\circ}$ or $40^{\circ} \mathrm{C}$, preceded by shaking chills. There is frequently mild to moderate elevation of the transaminases, although loss or reduction of the
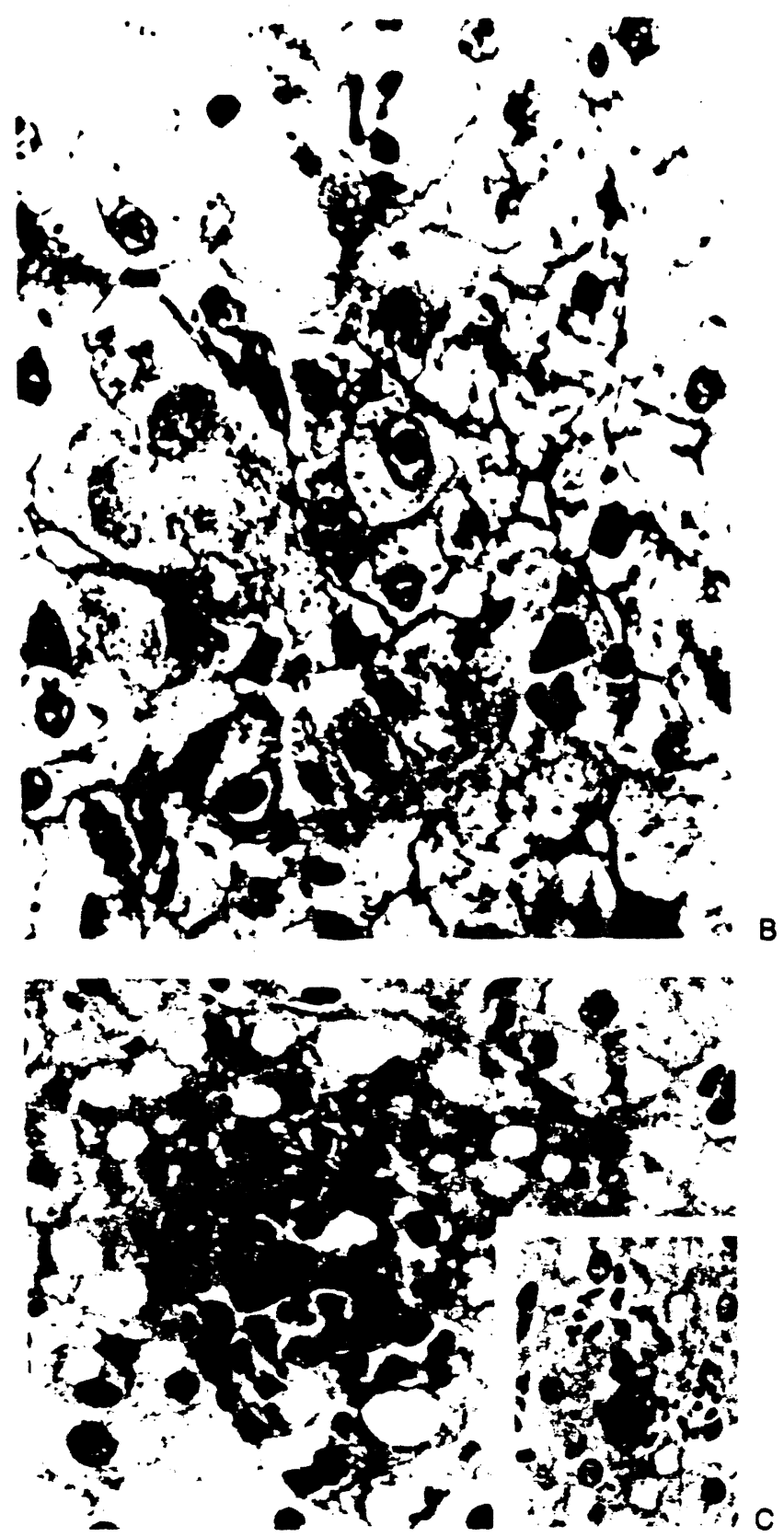

liver-based synthetic processes does not necessarily follow.

Thrombosis of the artery may lead to regional necrosis of the liver and abscess formation. Necrosis of the bile duct may also occur with leakage and bile peritonitis from which bacteria can be cultured. The most common offending organisms belong to the anaerobic and enteric groups.

The diagnosis of an arterial thrombosis can be made at an early time by keeping a high index of suspicion. A roentgenogram of the thorax may show gas-forming abscesses within the liver 
TABLE 2. Causes of Graft Ischemia

1. Unstable donor (h) potension, vasupressors)

2. Inadequate preservation

3. Prolonged cold ischemia

4. Prolonged uarm ischemia

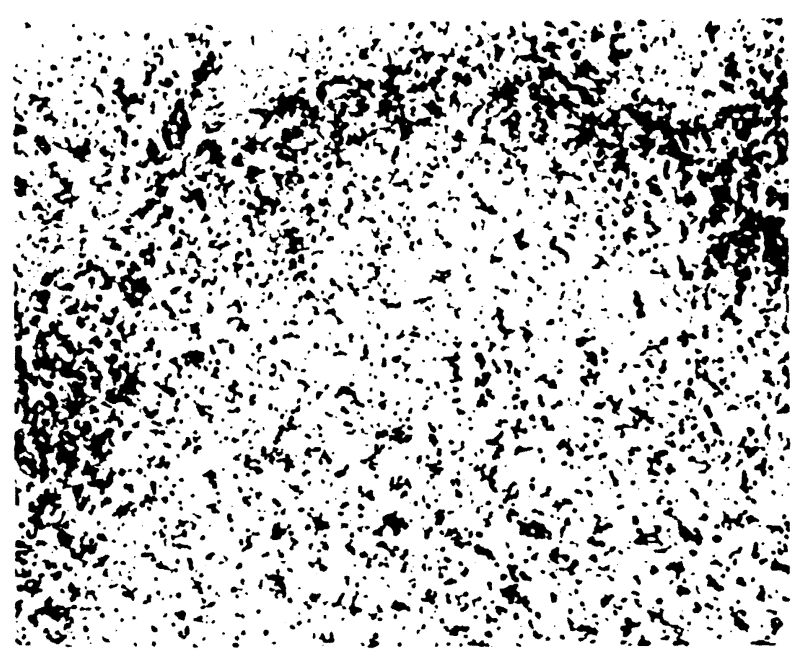

FIG. 3. Early harvesting injury. Injury in the earty period is characterized by centrilobular hepatocyte swelling and canalicular cholestasis. Periportal cells are preserved or even shrunken and acidophilic. Portal areas are inconspicuous (HEE, $\times 100$. )

parenchyma. An ultrasound or a computed tomography (CT) scan give additional information about the extent of the injury. A doppler ultrasound has been very useful in determining the patency of the artery and portal vein. If the artery cannot be seen to pulsate, a selective angiogram is mandatory. The treatment of choice is retransplantation, although a few patients have survived with dearterialized livers. The experience with this serious complication at the University of Pittsburgh is reviewed elsewhere in this issue of Seminars.

\section{INFECTION}

Bacterial, fungal, and viral infection can also lead to hepatic dysfunction following transplantation. $^{3,4}$ Viral infections may create especially serious problems in diagnosis and treatment, since they can infest and damage the grafts directly at variable and unpredictable times. Virus infections, of the herpes group (CMV, herpes simplex, EpsteinBarr virus, and herpes zoster) have been seen frequently. ${ }^{3}$ Attacks by the hepatitis virus usually occur 2 or 3 months after transplantation. The following cases will demonstrate some of the clinical features of these viral infections.

\section{Case 1: Varicella Infection}

OT 368, a 4-year, 10-month-old South American girl, underwent an orthotopic liver transplantation at the age of 4 years, 5 months. She had cirrhosis secondary to biliary atresia. She did well for 5 months until 5 days before her last admission when she entered a hospital in Caracas with a vesicular rash consistent with varicella. She was transferred to Pittsburgh for further treatment.

On physical examination, skin lesions of varicella were found mostly on the trunk and face, but also on the buttocks and right leg. The prothrombin time was 32.5 seconds with a control of 11.5 and the partial thromboplastin time was 58 seconds with a control of 26.5 seconds. The total and direct bilirubin were 5.3 and $3.7 \mathrm{mg} / \mathrm{dl}$, respectively. The SGOT was $5890 \mathrm{IU} /$; SGPT was $4389 \mathrm{IU} /$; ; alkaline phosphatase was $348 \mathrm{IU} / \mathrm{h}$; and gamma glutamyl transpeptidase (GGTP) was $629 \mathrm{IU} /$. The patient was hypotensive and in severe respiratory distress. Her course was one of rapid deterioration despite aggressive treatment and she died a few hours later. The autopsy revealed massive hepatic necrosis. Histologically, there were numerous intranuclear inclusion bodies in the periphery of the necrotic areas (Fig. 4). Varicella hepatitis has been described before as a cause of early ${ }^{5}$ and late $^{6}$ death after liver transplantation.

\section{Case 2: CMV Hepatitis}

OT 472, a 7-year-old white girl with biliary atresia, underwent an orthotopic liver transplantation on December 7, 1984. She developed severe rejection on the fourth postoperatie day, requiring treatment with increased steroids and monoclonal ALG (OKT3). Four weeks later, she developed a fever of $40.5^{\circ} \mathrm{C}$; marked elevation of the enzymes from previously normal values; increases of the total and direct bilirubin to 3.8 and $2.9 \mathrm{mg} / \mathrm{dl}$; and increased levels of SGOT, SGPT, and GGTP to 845,588 , and $534 \mathrm{IU} /$, respectively. CMV was isolated from urine, throat, bronchi, and blood. A liver biopsy revealed lobular hepatitis with multiple microabscesses. Numerous intranuclear and intracytoplasmic viral inclusions were noted similar to those in Figure 2. CMV was also isolated from the liver tissue. Treatment consisted of reduction of immunosuppression with a good response. The patient was discharged with normal liver function tests.

Cultures taken from throat washings, urine samples, blood buffy coats, and liver biopsies have aided in the diagnosis of these viral infections. 


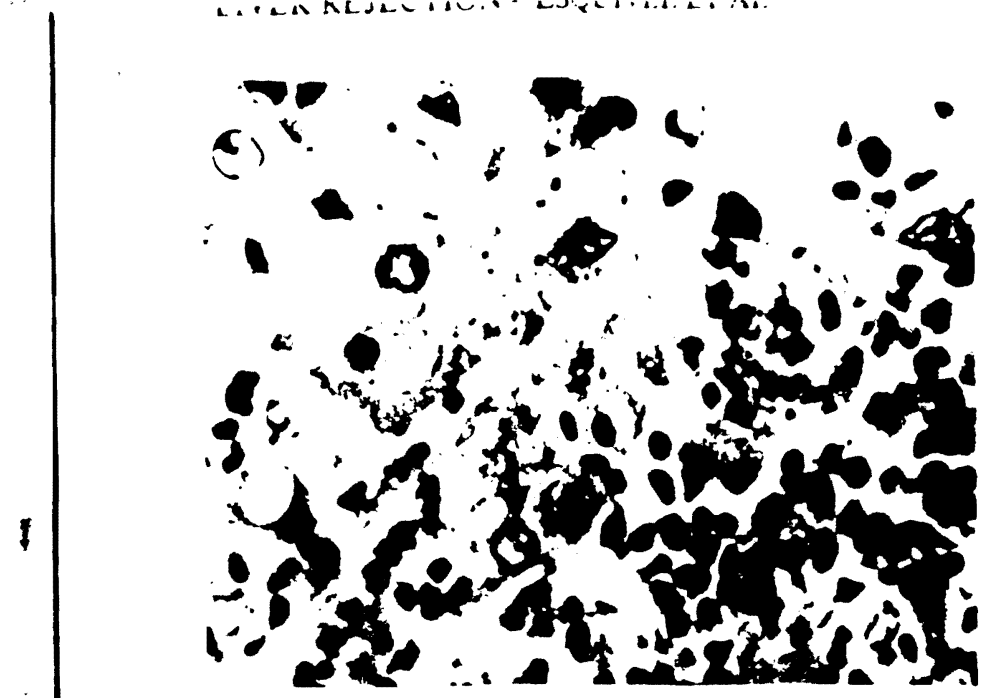

FIG. 4. Varicella hepatitis. At the margins of the necrotic zones, there were multinucleated hepatocytes and cells with waxy or eosinophilic intranuclear inclusions (arrow). (H\&E, $X$ 450.1

Fungal infections have been seen in liver transplant recipients and Candida has been the most common organism isolated. ${ }^{4}$ Septicemia with gram-negative and gram-positive organisms may also cause hepatic dysfunction.

\section{BILE DUCT COMPLICATIONS}

Complications with bile duct reconstruction used to be very common. Although these have been minimized with the standardization of reconstructive techniques, bile leak or biliary obstruction are important causes of hepatic dysfunction. ${ }^{7}$ An internal bile leak usually is manifested by acute peritonitis associated with mild elevations of the bilirubin and enzymes. Biliary leaks caused by bile duct disruption usually occur within 10 days after surgery. Drainage of bile may be present in the abdominal drains. T-tube cholangiogram, paraisopropyl iminodiacetic acid (PIPIDA) scan, and ultrasound are useful methods for making the diagnosis. The treatment consists of reoperation with repair of the bile duct disruption.

Stenosis of the bile duct is frequently associated with acute cholangitis characterized by fever, jaundice, and elevation of the transaminases. Bacteria indigenous to the gastrointestinal tract often can be cultured from the blood. Ultrasound may disclose dilated extrahepatic or intrahepatic bile ducts. The diagnosis is confirmed by percutaneous cholangiography or endoscopic retrograde pancreaticocholangiography (ERCP). This complication can usually be treated by intraluminal dilation via a percutane- ous catheter, but in some cases surgical correction is necessary.

\section{HEMOLYSIS}

Hemolysis has been observed in many patients after hepatic transplantation. At the University of Pittsburgh, approximately $17 \%$ of the patients received $\mathrm{ABO}$-unmatched transplants. These patients may develop hemolysis with an increase in the direct and indirect bilirubin associated with a decrease in the hematocrit value in the absence of hemorrhage. It has been well documented that liver grafts from donors having $A B O$ types different from the recipient can produce antirecipient $\mathrm{ABO}$ antibodies that have been detected from 8 to 16 days after transplantation and that persist for as long as $\mathbf{4 1}$ days postoperatively. ${ }^{8}$

\section{DRUG-INDUCED HEPATIC INJURY}

It is beyond the scope of this article to discuss all drugs responsible for hepatic dysfunction; in fact, with the advent of new sensitive tests, almost all drugs have been found to be associated with liver injury. The two mechanisms by which drugs can cause hepatic injury are direct toxicity and hypersensitivity. Most of the industrial agents responsible for liver injury do so by direct toxicity, that is, mushrooms and chlorophenothane (DDT). Halothane and phenytoin are drugs that may produce hepatic injury by hypersensitivity.

The diagnosis of a drug-induced injury is usually one of exclusion. In liver recipients, some of the most commonly used drugs that may be associated with hepatic dysfunction are listed in Table 3. A liver biopsy is usually not helpful, since the pattern of injury is variable and not specific. The hepatic dysfunction usually resolves when the offending drug is withdrawn.

Cyclosporine may cause hepatic dysfunction in renal allograft recipients. ${ }^{9}$ Approximately $20 \%$ (13 patients) of the 66 patients studied had at least one episode of hepatic dysfunction characterized by an elevation of bilirubin over $2.0 \mathrm{mg} / \mathrm{dl}$. Three of the these 13 cases had evidence of late toxicity that reversed after reduction of the dosages. In none of the renal patients with early reactions was the toxicity severe enough to warrant withdrawal of the drug. Hepatotoxicity in liver recipients is difficult to diagnosis and, in fact, an erroneous diagnosis may precipitate a rejection episode by an unwise reduction or discontinuance of the cyclosporine dose. 
TABLE 3. Frequently Used Drugs in Hepatic

Transplantation that May Be Associated with Hepatic Dysfunction

Immunosuppressants

Cyilosporine

Azathioprine

Antihypertensive and diuretic agents

Methyldopa

Hydralazine hydrochloride

Captopril

Clonidine hydrochloride

Ethacrynic acid

Antimicrobial agents

Penicillins (particularly synthetic compounds)

Cephalosporins

Erythromycin

Clindamycin

Aminoglycosides

Amphotericin B

Flucytosine

Trimethoprim-sulfamethoxazole

\section{SUMMARY}

Numerous causes can lead to hepatic dysfunction following orthotopic livertransplantation. The most common cause is rejection, which is usually nonpreventable. The clinical presentation, time of onset, and even treatment are variable. Other causes, such as perioperative ischemic injury, vascular thrombosis, and complications of bile duct reconstruction may be preventable with good surgical technique. Infections can also be minimized by careful adjustment of immunotherapy, avoidance overimmunosuppression, and the judicious use of antibiotics. Hepatic dysfunction following orthotopic lvier transplantation requires rapid assessment and proper treatment in order to prevent serious and possibly fatal complications.

Acknowledgment. Supported by Research Grants from the Veterans Administration and Project Grant No. AM-29961 from the National Institutes of Health, Bethesda, Maryland.

\section{REFERENCES}

1. Starzl TE, Iwatsuki S, Van Thiel DH, et al: Evolution of liver transplantation. Hepatology 2:614-636, 1982.

2. Tzakis AG, Gordon RD, Shaw BW Jr, et al: Clinical presentation of hepatic artery thrombosis after liver transplantation in the cyclosporine era. Transplantation. In press.

3. Ho M, Wajszczuk CP, Hardy A, et al: Infections in kidney, heart and liver transplant recipients on cyclosporine. Transplant Proc 15:2768-2772, 1983.

4. Wajszczuk CP, Ho M, Dummer JS, et al: Fungal infections in liver transplant recipients. Intern Med. In press.

5. Starz TE, Koep LJ, Porter KA, et al: Decline in survival after liver transplantation., Arch Surg 115:815-819, 1980.

6. Starz TE, Koep LJ, Halgrimson CG, et al: Fifteen years of clinical liver transplantation. Gastroenterology 77:375-388, 1979.

7. Iwatsuki S, Shaw BW Jr, Starzl TE: Biliary tract complications in liver transplantation under cyclosporine-steroid therapy. Transplant Proc 15:1288-1291, 1983.

8. Ramsey G, Nusbacher J, Starzl TE, Lindsay GD: Isohemagglutinins of graft origin after ABO-unmatched liver transplantation. N Engl J Med 311:1167-1170, 1984.

9. Klintmalm GBG, Iwatsuki S, Starzl TE: Cyclosporin-A hepatotoxicity in 66 renal allograft recipients. Transplantation 32:488-489, 1981.

10. Ware AJ, Luby JP, Eigenbrodt EH, et al: Spectrum of liver disease in renal transplant hepatitis. Gastroenterology 68: 755-764, 1975.

11. Starzl TE (with the assistance of CW Putnam): Experience in hepatic transplantation. Philadelphia, W.B. Saunders, 1969, pp 194-198.

12. Rodman JS, Deutsch DJ, Gutman SI: Methyldopa hepatitis. A report of six cases and review of the literature. Am J Med 60:941-948, 1976. 